\title{
Cronaca di un Convegno: per un nuovo umanesimo
}

Marco Strona

\section{Resumo}

Il testo fondamentale dell'Umanesimo è sicuramente l'Oratio de hominis dignitate di Pico della Mirandola, in cui, già dalle prime battute, viene proclamata la frase cardine del principio umanista: Magnum miraculum est homo.

La grandezza dell'uomo è "oggetto degno di contemplazione, è la dichiarazione di un valore che impone il rispetto; ma è altresì "oggetto che suscita un interrogativo: l'admiratio ricorda lo thaumazein aristotelico, la meraviglia da cui sgorga la domanda del "perché" e che muove la ricerca e genera la teoria".

La grandezza dell'uomo, che lo distingue da tutte le altre creature, non è data dalla quantità, ma è contrassegnata da un dono: quello della libertà.

La dignitas hominis, prosegue Capecci, "non sta nell'occupare un posto di preminenza o centrale nella gerarchia dell'universo, ma nella libertà": l'uomo, diversamente dall'animale, può, e deve, scegliere; scegliere fondamentalmente tra il finito e l'Infinito.

La libertà è connessa "al dinamismo insito nella creatura umana, che è il dinamismo dell'essere finito verso l'Infinito".

La dignità dell'uomo si fonda perciò nella libertà, in quanto solo questa può rappresentare la condizione della "realizzazione" dell'essere umano stesso, il suo sviluppo: ma l'autentica libertà si può dare solo di fronte a Colui che ha donato tale possibilità, cioè solo di fronte a Dio. 
È proprio questo il messaggio che S. Francesco ha voluto trasmettere all'uomo: solo di fronte a Dio siamo veramente liberi, possiamo chiamare tutti fratelli e sorelle e riconoscerci in loro come creature desiderose di condividere la vita con il Padre.

In un epoca come quella che stiamo attraversando, ha affermato Maria Clara Bingemer, "vivere umanamente è divenuto sinonimo di godimento delle delizie del consumismo: una certa deificazione della capacità consumistica è elevata allo status di reale religione".

L'incontro di Simone Weil, filosofa che ha vissuto pienamente le vicende tragiche del Novecento, con la figura di S. Francesco ha manifestato una "coerenza radicale"nel vivere e testimoniare valori esattamente opposti da quelli proposti dalla società del consumo.

Parole-chiavi: Nuovo Umanesimo, Liberta', Dignita' Dell’Uomo, Dio.

\section{Resumo}

O texto fundamental do Humanismo é, certamente, o Oratio de hominis dignitate de Pico della Mirandola, no qual, já desde as primeiras considerações, proclamou-se a frase cardeal do princípio humanista: Magnum miraculum est homo.

A grandeza do homem é "digno objeto de contemplação, é a declaração de um valor que impõe respeito"; mas é, além disso, "objeto que suscita uma interrogação: a admiratio lembra o thaumazein aristotélico, a maravilha da qual brota a pergunta sobre o 'porquê', e que move a procura e gera a teoria".

A grandeza do homem, que o distingue de todas as outras criaturas, não advém da quantidade, mas é diferenciada por um dom: o da liberdade.

A dignitas hominis, continua Capecci, "não está no ocupar um lugar de proeminência ou central na hierarquia do universo, mas na liberdade": o homem, à diferença do animal, pode e deve escolher; escolher fundamentalmente entre o finito e o "Infinito".

A liberdade está conexa "ao dinamismo inerente da criatura humana, que é o dinamismo do ser finito rumo ao Infinito".

A dignidade do homem fundamenta-se na liberdade, pois somente esta pode representar a condição da "realização" do próprio ser humano, o seu desenvolvimento: mas a autêntica liberdade pode acontecer somente diante d'Aquele que doou tal possibilidade, isto é, somente diante de Deus. 
Esta é a mensagem que São Francisco quis transmitir ao homem: somente diante de Deus somos verdadeiramente livres, podemos chamar a todos de irmãos e irmãs, e reconhecer-nos neles como criaturas desejosas de compartilhar a vida com o Pai.

Em uma época como a que estamos atravessando, afirmou Maria Clara Bingemer, "viver humanamente tornou-se sinônimo do prazer das delícias do consumismo: uma deificação da capacidade consumista foi elevada ao status de religião real".

O encontro de Simone Weil, filósofa que viveu plenamente os fatos trágicos do século XIX, com a figura de São Francisco, manifestou uma "coerência radical" no viver e testemunhar valores exatamente opostos aos propostos pela sociedade consumista.

Palavras-chave: Novo Humanismo, Liberdade, Dignidade do Homem, Deus.

Il giorno 30 aprile 2011 si è svolto nel convento di Monteripido il convegno internazionale Per un nuovo umanesimo: Francesco, S. Weil e la filosofia contemporanea, promosso e organizzato dalla Pastorale Universitaria di Perugia, dai Frati minori dell'Umbria, dall'Ufficio per i problemi sociali della diocesi di città di Castello e dall’Università degli studi di Perugia.

Il convegno ha rappresentato il compimento di un percorso formativo che ha coinvolto numerosi studenti di diverse facoltà, mediante incontri e tavole rotonde che si sono succedute durante quest'anno accademico, incentrate su tematiche riguardanti la persona, il rapporto tra l'uomo e l'ambiente nell'era globalizzata $\mathrm{e}$, in generale, sulla possibilità di ri-definire una nuova concezione di umanesimo dopo la "svolta" heideggeriana e sartriana.

I relatori del convegno sono stati il prof. Antonio Pieretti e il prof. Angelo Capecci, dell'Università di Perugia, il prof. Hernandez, dell'università Antonianum di Roma, il prof. Emmanuel Gabellieri, docente e preside della facoltà di filosofia dell’Università Cattolica di Lyon e la prof.ssa Maria Clara Bingemer, docente della Pontificia Università di Rio de Janeiro.

Il Prof. Hernandez ha trattato il tema dell'umanesimo in S. Francesco, illustrando brillantemente i tratti salienti della vita del "Poverello di Assisi", e cercando di individuare l'attualità del pensiero francescano soprattutto nell'ambito antropologico: la tematica della "fratellanza universale" è la cifra caratteristica della proposta di Francesco per la definizione di un umanesimo teocentrico e teologico. 
Il prof. Capecci, dopo aver esposto una sintesi storiografica del concetto di "umanesimo", ha illustrato il proprium di questo termine che risiede nella definizione di "categoria dello spirito".

I testo fondamentale dell'Umanesimo è sicuramente l'Oratio de hominis dignitate di Pico della Mirandola, in cui, già dalle prime battute, viene proclamata la frase cardine del principio umanista: Magnum miraculum est homo.

La grandezza dell'uomo, afferma Capecci, è "oggetto degno di contemplazione, è la dichiarazione di un valore che impone il rispetto"; ma è altresì "oggetto che suscita un interrogativo: l'admiratio ricorda lo thaumazein aristotelico, la meraviglia da cui sgorga la domanda del 'perché' e che muove la ricerca e genera la teoria".'

La grandezza dell'uomo, che lo distingue da tutte le altre creature, non è data dalla quantità, ma è contrassegnata da un dono: quello della libertà.

La dignitas hominis, prosegue Capecci, "non sta nell'occupare un posto di preminenza o centrale nella gerarchia dell'universo, ma nella libertà": '2 l'uomo, diversamente dall'animale, può, e deve, scegliere; scegliere fondamentalmente tra il finito e l'Infinito.

La libertà è connessa "al dinamismo insito nella creatura umana, che è il dinamismo dell'essere finito verso l'Infinito". ${ }^{3}$

La dignità dell'uomo si fonda perciò nella libertà, in quanto solo questa può rappresentare la condizione della "realizzazione" dell'essere umano stesso, il suo sviluppo: ma l'autentica libertà si può dare solo di fronte a Colui che ha donato tale possibilità, cioè solo di fronte a Dio.

È proprio questo il messaggio che S. Francesco ha voluto trasmettere all'uomo: solo di fronte a Dio siamo veramente liberi, possiamo chiamare tutti fratelli e sorelle e riconoscerci in loro come creature desiderose di condividere la vita con il Padre.

In un epoca come quella che stiamo attraversando, ha affermato la Bingemer, "vivere umanamente è divenuto sinonimo di godimento delle delizie del consumismo: una certa deificazione della capacità consumistica è elevata allo status di reale religione".

L'incontro di Simone Weil, filosofa che ha vissuto pienamente le vicende tragiche del Novecento, con la figura di S. Francesco ha manifestato una "coerenza radicale"nel vivere e testimoniare valori esattamente opposti da quelli proposti dalla società del consumo.

\footnotetext{
${ }^{1}$ A. Caprecci, Il pregiudizio storico, Città Nuova, Roma 2005, p. 107.

${ }^{2}$ Ibid, p. 111.

${ }^{3}$ Ibid, p. 113.
} 
La concezione weiliana della libertà si radica essenzialmente nella "mistica della povertà" che trova nel "Poverello di Assisi" il suo maggior rappresentante: la povertà che si rende visibile nello "spirito" implica, prosegue la Bingemer, "uno spirito di totale disponibilità e apertura fiduciosa a Dio e ai fratelli".

Il modello della povertà concepita in questi termini, come una "inclinazione esistenziale" e come una "regola di vita" è stata incarnata in maniera perfetta e unica da Gesù Cristo: è questo l'aspetto che ha affascinato e convertito la vita di Francesco di Assisi e che ha continuato sempre di più ad attrarre moltissime persone, tra cui anche Simone Weil.

Non è solo la povertà intesa come concetto astratto, ma è la povertà cristiana il segno indelebile dell'incontro tra la Weil e S. Francesco: si tratta, perciò, "della povertà che contiene purezza, bellezza e si trasforma in poesia $\mathrm{e}$ arte nella misura in cui è vissuta nella profonda radicalità".

La povertà diviene, in Simone Weil la condizione "per poter vivere liberamente e pienamente il contatto con la bellezza del mondo": lo spirito di povertà, per lei, comporta "una maggiore disponibilità a sentire e assaporare la bellezza del mondo, fatta di semplicità, purezza e verità"; ed è proprio tale "spirito di povertà" il cammino più sicuro verso la Verità.

La scelta della povertà conduce Simone Weil ad un contatto diretto con gli ultimi, i "minori" (ricordiamo l'esperienza fondamentale di Pòvoa de Varzim e quella della fabbrica tra gli operai) nei cui volti lei sperimenta in maniera diretta la presenza di Cristo: la scelta di "Madonna Povertà", permette a Simone la convivenza e la comunione con ogni essere umano, consentendo di sperimentare pienamente la bellezza della creazione in tutte le sue forme.

La bellezza per la creazione e lo stupore che si prova di fronte alle sue meraviglie è sostenuta e fondata dalla bellezza del Crocefisso: teologia della creazione e teologia della redenzione, fa brillantemente notare Gabellieri, trovano nel pensiero di Simone Weil un felice accordo.

Il "Cantico di Frate Sole", prosegue Gabellieri, mostra quale posto la bellezza del mondo può avere in un pensiero cristiano: "non solo il suo poema è poesia perfetta, ma tutta la sua vita fu poesia perfetta in azione".

La contemplazione, per Francesco, è sempre unita all'azione: questo è l'aspetto che più fece appassionare Simone Weil; in lei, fa notare Gabellieri, "l'unità tra azione e contemplazione, tra corpo e spirito non può essere sigillato in maniera totale che in un dono di sé": occorre, cioè, vedere in che modo la mistica della bellezza si articoli in Simone con la mistica della Croce (teologia della redenzione). 
Per giungere ad un'autentica scientia crucis, Simone Weil ricorre al concetto di "decreazione": l'Imitatio Christi deve passare attraverso una nostra personale Kenosis.

Una creatura ragionevole, afferma la Weil, "è una creatura che contiene in sé il germe, il principio, la vocazione della decreazione".

La proposta di Simone Weil al mondo d'oggi , afferma Gabellieri, riguarda una cristologia filosofica "che non sia una dissoluzione del Cristo della fede nel Verbo della filosofia, ma una manifestazione della convenienza dell'Incarnazione a riguardo della verità di Dio e dell'uomo: convenienza per la quale l'umiltà socratica trova il suo compimento nell'umiltà di Cristo, rivelazione di una saggezza suprema che è Amore".

Solamente partendo dagli "ultimi", dai poveri, mediante un processo kenotico di svuotamento, è possibile scoprire e ammirare la Bellezza, il vero volto di Cristo morto e Risorto: solamente da qui è possibile conoscere la "creazione come un tessuto di pensieri particolari di Dio", solamente da qui è possibile scoprire "il tutto nel frammento".

La sintesi francescana tra Eros, àgape e pathos, ripresa in maniera feconda da Simone Weil, permette perciò di inaugurare un "nuovo senso di umanesimo" fatto di compassione, apertura, relazione intima e comunione profonda con Dio padre; un nuovo umanesimo che deve sapersi tradurre anche, come ha affermato Pieretti, in praxis, in atteggiamento politico, rivolto cioè all'edificazione del Bene Comune.

Il tema dello sviluppo coincide infatti "con quello dell'inclusione relazionale di tutte le persone e di tutti i popoli nell'unica comunità della famiglia umana, che si costruisce nella solidarietà sulla base dei fondamentali valori della giustizia e della pace"

Marco Strona

Doutorando em Filosofia da Religião junto ao Pontificio Ateneo Santo Anselmo (Roma) Docente de Filosofia do Istituto Teologico Marchigiano di Ancona

Comunicação Recebida em 15/08/2011

Comunicação Aprovada em 28/10/2011

${ }^{4}$ Benedetto XVI, Caritas in Veritate, $n^{\circ} 53$. 